\title{
Organización del sistema de reglas de la tilde en el español: fundamentos lingüísticos
}

\author{
Organization of the orthographic accent rule system in Spanish: Linguistic \\ Foundations
}

\author{
Luis Alberto Mamani Quispe \\ Universidad Nacional Mayor de San Marcos, Lima, Perú \\ Universidad de Ciencias y Humanidades, Lima, Perú \\ Contacto: 1mamaniq@unmsm.edu.pe \\ https://orcid.org/0000-0002-3781-8688
}

\begin{abstract}
Resumen
Tras mostrar que las reglas vigentes del acento gráfico en español exhiben incoherencias e inconsistencias, en el presente trabajo se plantea una manera diferente de organizarlas. Para desarrollar la propuesta se ha recurrido a la teoría de los sistemas complejos debido a que las normas del acento gráfico están incardinadas en el sistema de escritura del español y a que se relacionan con unidades del sistema lingüístico. Ello nos ha permitido mostrar que la organización de las reglas no puede realizarse al margen del ordenamiento de las palabras. Teniendo como base esto último se propone cuatro clases de tildes sin reglas generales, sin excepciones y sin reglas de carácter prohibitivo. La principal consecuencia, en términos de su utilidad, es que la enseñanza de la tilde no puede empezar por las reglas generales, sino por las palabras con hiato.
\end{abstract}

Palabras clave: Acento gráfico; Teoría de sistemas complejos; Enseñanza de la tilde

\begin{abstract}
After showing that the current orthographic accent rules in Spanish exhibit incoherencies and inconsistencies, this paper proposes a different way of organizing them. Due to the fact that the orthographic rules are embedded in the Spanish writing system and that they are related to the linguistic system units, the complex system theory has been used to develop this proposed organization. This has allowed us to show that the organization of the rules cannot be done without taking into account the word order. Based on the latter, four classes of accent marks are proposed, without general rules, without exceptions and without rules of a prohibitive nature. The main consequence, in terms of its usefulness, is that the teaching of the orthographic accent use cannot begin with the general rules, but with the words that have hiatus
\end{abstract}

Keywords: Orthographic accent; Complex system theory; Written accentuation teaching

Recibido: $12.02 .17 \quad$ Aceptado: 18.02 .18 


\section{Introducción}

Un tipo de error sistemático en el empleo de la tilde por parte de quienes han iniciado o van a iniciar sus estudios universitarios acontece en las palabras que presentan el hiato que Portilla (2003) denomina acentual (avería, increíble, etc.). Parece que se incurre en este error no solo en España (Pujol, 2005) y Perú (Porti1la, 2003), sino en otros países de habla hispana porque la tilde en estas palabras supone una contradicción a las reglas generales; así, el hablante se vería obligado a realizar operaciones de gestión de la información (Mamani, 2013).

Esa sistematicidad estaría vinculada a la homogeneidad con que se programan los contenidos de dicho tema en la escuela obligatoria hispánica. Si bien algunos currículos no muestran información sobre tal aspecto, los de Chile (Mineduc, 2012; 2016), Ecuador (Ministerio de Educación, 2016) y Perú (Minedu, 2009) exhiben casi la misma secuencia: reglas generales, reglas excepcionales, tilde diacrítica y cuestiones periféricas (tilde en extranjerismos, mayúsculas, etc.). Esta misma sucesión se halla en los manuales de ortografía.

El origen de esta uniformidad en la secuencia de contenidos es el ceñimiento a la forma en que la Real Academia Española y la Asociación de Academias de la Lengua Española (RAE \& ASALE, 2010) presentan las normas del acento gráfico: reglas generales, tilde en las palabras con secuencias vocálicas, tilde diacrítica y casos marginales. Y lo que origina este orden es la certeza de la existencia de reglas generales.

La primera regla general establece que los monosílabos se escriben sin tilde. Como es obvio, esta norma afecta solo a los monosílabos, no a las palabras en general. Por tanto, esta regla está en las mismas condiciones que aquella que señala que se tilda las palabras con hiato acentual. Por otra regla general, se escriben con tilde las palabras agudas terminadas en vocal o en vocal seguida de $<\mathrm{n}>$ $\mathrm{o}<\mathrm{s}>$. En análoga condición se hallan las reglas, también denominadas generales, de las palabras graves, esdrújulas y sobreesdrújulas. Como es evidente, estas normas comprenden solo a un tipo particular de palabras, por lo cual, no podría corresponderles la denominación de generales. Por ello, se puede afirmar que en el sistema de reglas del acento gráfico (en lo sucesivo SRAG) vigente las reglas generales no parecen gozar de tal estatus. 
Esa primera regla general, además, es prohibitiva: los monosílabos no se tildan (RAE \& ASALE, 2010). También son prohibitivas las normas de la tilde diacrítica que determinan que no se tildan las palabras que son cuasihomófonas de las formas que sí se escriben con esta marca (quién, dónde, cómo, etc.). El problema con este tipo de reglas es que se contradice el propósito del SRAG: identificar en la escritura la sílaba tónica.

Por otra parte, en las reglas de la tilde identificamos dos asimetrías: i) solo a una pequeña cantidad de monosílabos y de polisílabos le corresponde acento gráfico; pero para los primeros se ha creado tanto una regla general que indica ello como algunas normas específicas en la tilde diacrítica que señalan qué monosílabos no se tildan; en contraste, para los polisílabos no se ha creado ninguna regla para ello, ni general ni específica. ii) Lo excepcional de la tilde en algunos monosílabos ha dado lugar a la creación de la tilde diacrítica; pero no se ha creado una clase de tilde para los polisílabos con hiato acentual (día, raíz, etc.) que se tildan como excepción a las normas generales.

Lo expuesto nos permite afirmar que el modo en que el SRAG está organizado no es el más adecuado. Si el criterio cuantitativo expresado en que las reglas generales "afectan a todo un ámbito de la escritura" (RAE \& ASALE, 2010, p. 11) es inadecuado para organizar las reglas, lo crucial es la identificación del criterio pertinente.

Si el propósito del SRAG es indicar en la escritura la ubicación del acento prosódico (RAE \& ASALE, 2010), entonces sus reglas se limitan a señalar las características que deben cumplir determinadas palabras para poder ser escritas con tilde. Las normas, por su naturaleza, hacen referencia a unidades lingüísticas, no las crean. Es decir, hay palabras agudas, graves o esdrújulas no porque el SRAG lo declare, sino porque existe un sistema en el que dichas unidades son admitidas con tales propiedades. Por ello, el ordenamiento del SRAG no puede ser ajeno a la organización de las unidades con las que se vincula. La cuestión es determinar cómo se pueden vincular ambos ordenamientos. La lingüística nos proporciona información acerca de las maneras en que están organizadas las unidades lingüísticas en su sistema, pero ello no tiene una automática proyección en la organización de las normas. Por esa razón, debemos recurrir a algún medio que 
nos permita establecer esta relación. Por los resultados que se han conseguido en algunos ámbitos diferentes al que aquí nos ocupa, en esta labor nos pueden ser de utilidad los aportes de la teoría de sistemas complejos (Ramírez, 2014).

Si la suposición de que el SRAG tiene reglas generales y excepcionales que han determinado la programación de su enseñanza en la escuela obligatoria, un ordenamiento diferente de dichas reglas, con el fundamento respectivo, tendría como consecuencia una programación distinta, sobre todo si no se postulan reglas generales. Los beneficiados directos de los resultados de este trabajo serían los aprendices de la normativa, debido a que un SRAG óptimamente organizado sería más fácil de aprender que uno inconsistente o incongruente.

\section{Marco de referencia}

\subsection{Antecedentes}

No hemos podido conocer trabajos académicos que hayan abordado explícitamente la manera en que está organizado el SRAG. Debido a que vamos a cuestionarlo, haremos explícito el ordenamiento que proponen la RAE y la ASALE (2010). La forma en que presentan las normas no permite advertir su organización interna, por tanto, para establecerla se identificarán las clases de palabras que están incluidas en cada norma y luego se determinará el criterio según el cual pueden ser agrupadas. Así, las palabras comprendidas en las reglas generales exhiben alguna de estas características: agudas, graves, esdrújulas, sobresdrújulas, con diptongo, con triptongo, con hiato, los adverbios que culminan en -mente y los monosílabos. Respecto de estas reglas, se admite la existencia de dos tipos de excepciones: i) las palabras con hiato acentual; y ii) algunos monosílabos y polisílabos a los que les corresponde la tilde diacrítica. Entonces, se puede afirmar que en el SRAG vigente hay tres clases de tildes: i) reglas generales; ii) tilde diacrítica; $y$, iii) una tilde sin nombre, que se aplica en las palabras con hiato acentual. La primera de estas tildes abarca normas de dos tipos: i) positivo; ii) prohibitivo. Las normas positivas son cuatro, cada una de las cuales regula la tilde en las siguientes clases de palabras: a) agudas; b) graves; c) esdrújulas y sobresdrújulas; y d) adverbios terminados en -mente. La regla prohibitiva es la que señala que los monosílabos se escriben sin tilde. 
La tilde diacrítica incluye también reglas de ambos sentidos: positivo y negativo. Las primeras regulan la tilde de la conocida lista de monosílabos y las palabras interrogativas y exclamativas. Las prohibitivas corresponden a los pares cuasihomófonos de las palabras interrogativas y exclamativas (v. g. los relativos "que", “donde"; la conjunción "que”, etc.).

La tercera clase de tilde, la innominada, presenta una sola regla en sentido positivo: se tilda la vocal cerrada de los hiatos acentuales.

\subsection{La teoría de los sistemas complejos}

El enfoque sistémico centra su óptica en buscar las relaciones entre elementos que aparentemente se presentan desvinculados. Emerge como un esfuerzo por superar las limitaciones de los enfoques analítico-reduccionistas y sus principios mecánico-causales (Arnold, 1998; Echeverría, 2010; Earls, 2011). Rodríguez (2011) identifica tres perspectivas en los estudios de la complejidad: i) ciencias de los sistemas complejos; ii) pensamiento complejo; y iii) teoría de los sistemas complejos.

El planteamiento de García (2006), la teoría de los sistemas complejos (en lo sucesivo TSC), es una metodología de trabajo interdisciplinario y a la vez un marco conceptual que fundamenta sobre bases epistemológicas dicho trabajo. García (2006; 2011) identifica dos tipos de sistemas: i) descomponibles, cuyas partes pueden ser aisladas y modificadas independientemente unas de otras; ii) complejos, cuyos elementos están organizados por niveles con dinámicas propias, pero interactuantes, lo que causa que cambien por reorganizaciones sucesivas. Un sistema complejo es inevitablemente una construcción del sujeto (Echeverría, 2010; Duval, 2014). Es una propuesta de organización de un recorte de la realidad. El investigador selecciona las situaciones, los fenómenos o los procesos y conforma con todo ello una entidad que tiene un funcionamiento especial. En esta labor de selección, el científico es guiado por las preguntas a las que pretende dar respuesta en su investigación (García, 2006; Duval, 2014).

La identificación y la selección de "datos”, que proveerán el soporte empírico de su estudio, estarán determinadas por dos elementos: i) el marco epistémico: 
cómo define los objetivos de su investigación orientados por el tipo de preguntas a las cuales intenta responder; ii) de dominio empírico: datos de la experiencia que serán privilegiados por la investigación, en virtud de su relación con las propias concepciones del investigador.

Sobre la naturaleza epistemológica de su perspectiva, García (2006) afirma que ningún sistema está dado en el punto de partida de la investigación. Por ello, la definición adecuada de un sistema solo puede surgir en el transcurso de la propia investigación y para cada caso particular. Y acerca de los fundamentos empíricos de su propuesta señala que no se aceptará la explicación del funcionamiento de un sistema si las constataciones empíricas la refutan.

El procedimiento de trabajo que propone García (2006) comprende las siguientes fases: i) reconocimiento general de los problemas que se procura interpretar y para los cuales se intenta encontrar solución; ii) análisis de estudios anteriores realizados sobre aspectos diversos de dicha problemática; ii) identificación de elementos y relaciones para caracterizar, en primera aproximación, un sistema que involucre la problemática referida en las fases 1 y 2 ; iv) planteamiento de hipótesis de trabajo; v) identificación de la problemática a investigar en cada subsistema para verificar o refutar las hipótesis sobre sus funciones dentro del sistema; vi) investigaciones disciplinarias de los problemas identificados; vii) primera integración de los resultados que se obtuvieron en la fase vi; viii) repetición de las fases v y vi en relación con la nueva definición del sistema; ix) segunda integración de resultados y nueva redefinición del sistema; $x$ ) repetición sucesiva de las fases viii y ix tantas veces como sea necesario hasta llegar a una explicación coherente que dé cuenta de todos los hechos observados y responda a las preguntas que han ido surgiendo en el proceso descrito.

\section{Propuesta de organización del SRAG}

\subsection{Justificación de un abordaje individual}

Alegamos tres razones para la viabilidad de la TSC en la presente investigación, que se ha iniciado sin la intervención de un equipo multidisciplinario: i) los estudios sectoriales pueden ser individuales (García, 2006); ii) lo característico de un sistema 
complejo, las propiedades emergentes, no está reñido con una investigación unidisciplinaria; y iii) hay trabajos individuales de índole lingüística, como los realizados por Martínez (2010) o López (2013), sin coordinaciones multidisciplinarias en sus inicios.

\subsection{Caracterización de los sistemas}

El SRAG, por ser creado, no tiene dinámica propia y tampoco presenta propiedades emergentes. No es un sistema complejo, sino descomponible (García, 2006); así, sus constituyentes, tras proporcionar su aporte al sistema del cual forman parte, no generan en este el surgimiento de propiedades cualitativamente diferentes a ellos. Es decir, la suma de reglas solo genera un conjunto de reglas. A pesar de ello, persistiremos en la TSC por lo siguiente: i) con este marco hay sustento teórico para relacionar el ordenamiento del SRAG con la ubicación que tiene en el sistema de escritura; ii) debido a que el SRAG es un sistema artificial, su organización es inconcebible al margen del ordenamiento de las unidades lingüísticas con las que se vincula; y iii) las reglas de la tilde se relacionan con unidades que pertenecen al sistema lingüístico, que es complejo.

En lo referente al sistema lingüístico, Baicchi (2015) señala que es un sistema complejo adaptativo. Cada componente (fonológico, morfológico, etc.) tiene una dinámica propia y lo que emerge de su interacción es algo cualitativamente diferente de cada uno de ellos. Por otra parte, el sistema como una totalidad se adapta a los cambios diacrónicos que experimentan sus componentes, cuyas dinámicas son diferentes. Entonces, al presentar propiedades emergentes y reestructuraciones sucesivas, es complejo.

\subsection{EI SRAG como parte de la escritura}

El SRAG forma parte del sistema de escritura de la lengua española. Su rol es permitir representar el acento de algunas palabras. Por otra parte, si se tiene en cuenta que ha sido creado para ser aprendido, el SRAG se relaciona con el sistema cognitivo del aprendizaje. Al ser esta su condición, debe cumplir dos exigencias: i) ser lo más asequible posible al sistema del aprendizaje; ii) representar lo más fielmente posible las unidades lingüísticas respectivas. No se debe olvidar que el 
sistema lingüístico y el del aprendizaje están en posesión del hablante, mientras que el SRAG, como parte del sistema de escritura, tiene carácter exógeno.

El sistema de unidades lingüísticas con el que el SRAG se relaciona no está identificado. Saber que la tilde representa el acento de algunas palabras es apenas una orientación, pues al ser un suprasegmental, debe coaparecer siempre con otras unidades, y la ubicación exacta de estas se desconoce. Ello contrasta con lo que ocurre con el sistema de grafemas (SIGRA en lo que sigue), pues se sabe que se vincula con los fonemas segmentales; y este saber permite hablar de una relación de emparejamiento, incluso en casos de poligrafía, ya que un fonema está asociado a un grafema. Esto difiere de lo que ocurre en el caso de la tilde: i) es excepcional que a un acento le corresponda una tilde; ii) el acento es un suprasegmental, por lo que debe aparecer asociado a otras unidades lingüísticas; iii) para registrar la tilde de algunas palabras, se requiere considerar necesariamente el SIGRA; y iv) para poder aprender el SRAG inevitablemente se tuvo que haber aprendido el SIGRA. Por lo señalado, se podría afirmar que aprender el SRAG difiere de aprender el SIGRA.

\subsection{EI SRAG y el sistema lingüístico}

Silva-Corvalán (2001) y Moreno (2008) nos recuerdan que una lengua no es un sistema real, es una idealización. Esta construcción teórica sirve para aglutinar una serie de dialectos que tienen entre sí determinadas semejanzas gramaticales. Un dialecto sí puede considerarse un sistema lingüístico, pues comprende un sistema fonológico (inventario de fonemas y sus posibilidades combinatorias), un sistema morfológico (morfemas y sus combinaciones), etc. Un hablante adquiere un dialecto y es con este que va a aprender el SRAG; tendrá menos o más dificultades según posea uno u otro dialecto: si las reglas involucran la letra $<_{\mathrm{S}}>$, los hablantes seseantes y ceceantes tendrán más dificultades para dominar el SRAG. Entonces, la escritura es la representación gráfica de dialectos semejantes.

\subsection{Los sistemas implicados en las reglas}

Al ser natural y hallarse en la mente del hablante, el sistema lingüístico no puede interactuar con el SRAG. Al haber sido creado para regular la representación gráfica del acento, el SRAG debe poder enlazarse con las unidades lingüísticas que 
lo portan. La manera en que ello se logra es mediante las reglas; y debido a que estas son enunciados, el vínculo se consigue a través de menciones a las unidades que van a ser consideradas en la normativa. Y no hay que olvidar que para la tilde la unidad de referencia es la palabra escrita, entonces la relación con el sistema lingüístico es a través de un sistema en el que se regula cómo se escriben las palabras. Entonces, el primer sistema con el que el SRAG se vincula es aquel donde se regula la conformación de la palabra escrita (SIPAE en lo que sigue).

En cuanto a las menciones de las reglas, algunas se refieren a unidades identificables por el lugar en el que presentan el acento: agudas, graves, esdrújulas y sobresdrújulas. Esto supone reconocer la existencia de un subsistema en el que estas son admitidas con tales propiedades. Ese subsistema sería aquel donde acontece la asignación del acento (Núñez \& Morales-Front, 1999; RAE \& ASALE, 2011; Hualde \& Colina, 2014); el mismo donde, según la conformación de la palabra, se tiene que optar por una de las tres ventanas de acentuación. En este subsistema, las unidades ya han experimentado sus procesos de formación y cuentan con las marcas de concordancia respectivas. Se trata de palabras morfosintácticas (Radford, Atkinson, Britain, Clahsen, \& Spencer, 2000) (SIPA en lo sucesivo). Pero en el SIPA, no hay palabras sobresdrújulas (Núñez \& Morales-Front, 1999; Hualde \& Colina, 2014); esas unidades solo existen en la escritura porque la RAE y la ASALE (2010) han establecido que los enclíticos se escriban unidos a la forma verbal con la que coaparecen. Entonces, las sobresdrújulas pertenecen al SIPAE. Por otra parte, en el SIPA hay unidades sin acento, y tales unidades no pueden ingresar al SRAG pues su propósito es regular la representación gráfica del acento. Entonces, el sistema con el que el SRAG se relaciona exactamente es solo una parte del SIPA: el sistema de palabras con acento (SIPACA en lo que sigue).

Otras reglas de la tilde se refieren a la pertenencia de las palabras a ciertas categorías: pronombres personales, palabras interrogativas, palabras exclamativas, etc. Entonces la relación con el SIPACA se consolida, pues todas sus unidades portan este tipo de información. Se debe mencionar que la asignación del acento toma en cuenta la categoría gramatical (RAE \& ASALE, 2011; Hualde \& Colina, 2014). 
Finalmente, al tener reglas que mencionan letras, el SRAG debe conectarse con el SIGRA. Esta relación no es directa porque indicar que solo se tilda las agudas y graves que culminan en determinadas letras supone primero un enlace con el SIPACA y recién desde allí, otro con el SIGRA. Todo lo indicado se puede apreciar en la figura 1 .

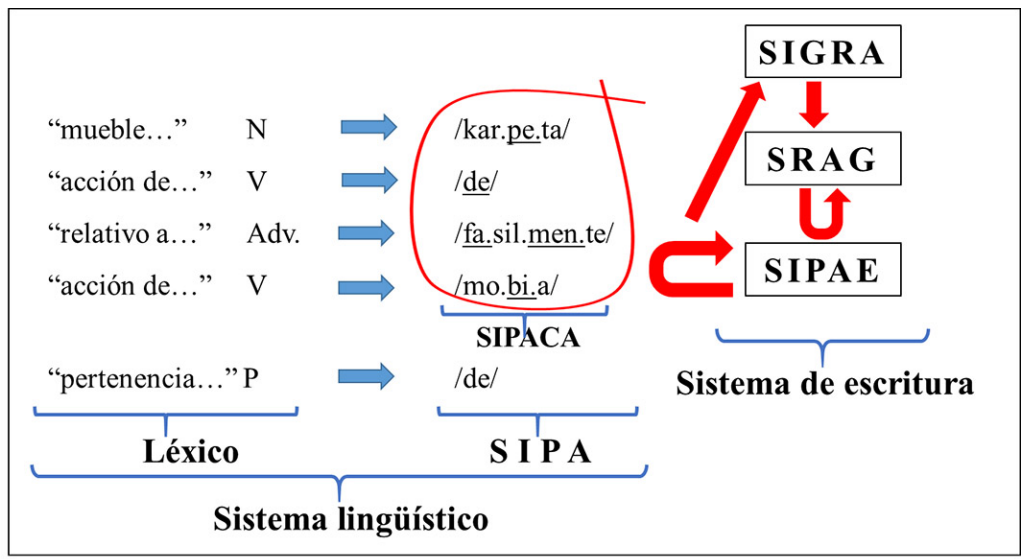

Figura 1. Sistemas con los que se relacionan las reglas de la tilde

Fuente: Elaboración propia

\subsection{La información de las reglas}

Determinar cómo está organizado el SIPACA exige recurrir al modo en que las normas se han enunciado, pues la única manera en que alguna palabra puede ingresar al SRAG es a través de las menciones de su normativa. Ordenará nuestra tarea la noción de coherencia textual de De Beaugrande y Dressler (2005): las configuraciones de los conceptos y las relaciones que subyacen al texto son accesibles entre sí e interactúan de modo relevante. Entendemos que por esta exigencia toda norma del SRAG debe contener obligatoriamente información acerca del acento. A su vez, una regla será coherente si codifica dos tipos de información: i) la que se refiere al total de unidades válidas para la norma (por ejemplo, todas las palabras agudas, todas las graves, etc.); y ii) la que señala a qué unidades de ese total les corresponde tilde (v. g. de todas las agudas, solo las terminadas en vocal o 
en vocal seguida de $<$ n $>$ o $<$ s $>$ ). Es decir, si una regla establece a qué palabras les corresponde tilde, ello supone necesariamente alguna clase de totalidad a la cual pertenecen dichas palabras. Está claro que la cantidad de unidades comprendidas en el segundo tipo de información no puede ser mayor a la del primer tipo.

En cuanto a la información del primer tipo se debe indicar que la sumatoria de cada total de cada norma da por resultado el universo de unidades válidas para el SRAG; si una palabra no está descrita en dicha información, no entra a él. Por otro lado, por el segundo tipo de información obtenemos el total de palabras que serán tildadas. Por ello, la información que emplearemos para ordenar el SIPACA será la del primer tipo. No consideraremos las reglas prohibitivas, pues no logran establecer ninguna conexión.

\subsection{Universo de palabras que ingresa al SRAG}

En este apartado se extraerá de cada regla la información correspondiente al primer tipo y luego se discutirá su pertinencia en la organización de las unidades respectivas.

Para empezar, la tilde diacrítica actual hace referencia a dos tipos de palabras: monosílabos y polisílabos interrogativos y exclamativos. Entonces, tanto el monosilabismo como la pertenencia a estas categorías serán las primeras características a ser consideradas en la organización de las palabras. Es decir, ingresan al SRAG los monosílabos con acento y los polisílabos interrogativos y exclamativos, y estos últimos no por ser graves, sino por su adscripción a estas dos categorías.

Las palabras agudas son el universo de la segunda norma, y así entran al SRAG. Lo mismo sucede con las palabras graves, que constituyen la totalidad de unidades de la tercera regla. También ingresan todas las esdrújulas y sobresdrújulas, pues conforman el total de expresiones de la cuarta regla, aunque estas últimas no son tomadas del SIPACA. Asimismo, el hiato acentual es otra propiedad relevante para la conformación de otra totalidad; o sea, toda palabra que lo presente ingresa al SRAG por tal condición, no por ser aguda o llana. Finalmente, existe una regla cuyo total de unidades son los adverbios terminados en -mente, 
que ingresan al SRAG por presentar dos acentos, pues para decidir su tilde se los descompone en dos constituyentes y se somete al primero a las reglas generales.

A diferencia de lo que ocurre con los monosílabos, no hay en las normas una mención explícita a los polisílabos, pero la referencia a las agudas, llanas, etc., supone considerar su naturaleza polisilábica. Lo mismo se puede decir de las palabras con hiato acentual y de las unidades con dos acentos. Esto quiere decir que el SIPACA, según demandas del SRAG, está organizado en dos grandes grupos: i) monosílabos; y ii) polisílabos.

El grupo de monosílabos no puede ser dividido, pues en el SIPACA solo hay unidades con acento. En el grupo de polisílabos, ello sí es necesario pues algunos ingresan por ser agudos, otros por ser graves, etc. Sobre ello, lo común a las palabras agudas, graves y esdrújulas es que exhiben solo un acento principal; en consecuencia, el criterio para haberlas clasificado de esta manera es el lugar del acento. Estas palabras conforman un grupo distinto del que está compuesto por las que tienen dos acentos, unidades inclasificables por el lugar del suprasegmental. Entonces, entre los polisílabos hay que diferenciar entre estos dos grupos de palabras. Por lo tanto, el número de acentos es un criterio pertinente para la clasificación de las palabras según las exigencias del SRAG.

Por otra parte, si hay polisílabos que ingresan al SRAG por ser interrogativos o exclamativos, entonces en el grupo de polisílabos con un acento se debe separar este grupo de aquel otro constituido por unidades clasificables por el lugar en el que lo presentan.

La existencia de una regla cuya totalidad de unidades son las palabras con hiato acentual obliga a realizar otra división al interior de los polisílabos con un acento: por un lado, los que presentan este tipo de hiato y, por otro, los que carecen de él.

Entonces, el SIPACA se organiza según los criterios siguientes: número de sílabas, número de sílabas tónicas, lugar de la sílaba tónica, ocurrencia del hiato acentual y adscripción a las clases de palabras interrogativas o exclamativas. La figura 2 lo ilustra. 


\begin{tabular}{|c|c|c|c|}
\hline \multicolumn{4}{|c|}{ Organización del SIPACA } \\
\hline \multicolumn{4}{|l|}{ Monosílabos } \\
\hline \multirow{6}{*}{ Polisílabos } & \multirow{5}{*}{ Con un acento } & \multicolumn{2}{|c|}{ Interrogativos y exclamativos } \\
\hline & & \multicolumn{2}{|c|}{ Con hiato acentual } \\
\hline & & \multirow{3}{*}{$\begin{array}{l}\mathrm{Ni} \text { interrogativos ni } \\
\text { exclamativos y sin } \\
\text { hiato acentual }\end{array}$} & Agudos \\
\hline & & & Graves \\
\hline & & & Esdrújulos \\
\hline & Con dos & entos & \\
\hline
\end{tabular}

Figura 2. Organización del SIPACA según los rasgos que considera el SRAG Fuente: Elaboración propia

\subsection{Propuesta de organización del SRAG}

\subsubsection{Condiciones y criterios}

Cada regla que se proponga presentará los dos tipos de información señalados: i) total de palabras pasibles de aplicación de la regla (TOP en lo sucesivo); ii) requisitos que deben cumplir las unidades, de ese total, para ser escritas con tilde; es decir, la condición para la tildación (CT en lo que sigue).

El SIPAE presenta grupos terminales, que pueden verse en el extremo derecho de la figura 3. Cada grupo terminal será el TOP de una regla. Ninguna unidad del SIPAE puede quedar fuera del SRAG. Por lo dicho, si cada grupo terminal de palabras es el TOP de una regla y esta se limita a señalar cuáles de ese total deben ser escritas con tilde, es imposible que se pueda originar una regla de tipo prohibitivo.

\begin{tabular}{|c|c|c|c|}
\hline \multicolumn{4}{|c|}{ Organización d e l S I PAE } \\
\hline \multicolumn{4}{|l|}{ Monosílabos } \\
\hline \multirow{7}{*}{ Polis ílabos } & \multirow{6}{*}{ Con un acento } & \multicolumn{2}{|c|}{ Interrogativos y exclamativos } \\
\hline & & \multicolumn{2}{|c|}{ Con hiato acentual } \\
\hline & & \multirow{4}{*}{$\begin{array}{l}\mathrm{Ni} \text { interrogativos } \mathrm{ni} \\
\text { exclamativos y sin } \\
\text { hiato acentual }\end{array}$} & Agudos \\
\hline & & & Graves \\
\hline & & & Esdrújulos \\
\hline & & & Sobresdrújulos \\
\hline & \multicolumn{3}{|c|}{ Con dos acentos } \\
\hline
\end{tabular}

Figura 3. Organización del SIPAE según los rasgos que considera el SRAG Fuente: Elaboración propia 
Por otra parte, la existencia de estos grupos terminales de palabras muestra que en el SRAG no puede haber reglas generales: si cada grupo terminal es el TOP de una regla, dicho grupo aglutinará siempre un conjunto de propiedades particulares. La única posibilidad de postular una regla general es que su TOP comprenda todo el universo de palabras y su CT incluya todas las que reciben tilde. En ese caso, el SRAG tendría una única regla.

En lo referente a las excepciones a las reglas, se puede afirmar que emergerán solo si, paradójicamente, toman en su TOP unidades que rebasan su marco de regulación. Ese sería el caso, en el actual SRAG, de la "regla general" que admite como TOP a todas las palabras llanas; esta norma indica en su CT que se escriben con tilde las que terminan en secuencia consonántica o en cualquier consonante menos $<\mathrm{n}>\mathrm{o}<\mathrm{s}>$. Sin embargo, sin cumplir estas condiciones, se tilda las graves que presentan hiato acentual y las palabras interrogativas y exclamativas. Esto quiere decir que en el TOP de esta norma no pueden estar todas las graves.

\subsubsection{Inventario de reglas}

Si sabemos que cada grupo terminal le corresponde a una regla, entonces tendríamos las siguientes: primera, para todos los monosílabos; segunda, para los polisílabos interrogativos y exclamativos; tercera, para las palabras con hiato acentual; cuarta, para las agudas; quinta, para las graves; sexta, para las esdrújulas; séptima, para las sobresdrújulas; y octava, para los adverbios en -mente. La figura 4 ilustra lo dicho.

\begin{tabular}{|c|c|c|c|c|}
\hline \multicolumn{4}{|c|}{ Organización de I SIPAE } & RE GLA S \\
\hline \multicolumn{4}{|c|}{ Monosílabos } & PRIMERA \\
\hline \multirow{7}{*}{ Polisílabos } & \multirow{6}{*}{ Con un acento } & \multicolumn{2}{|c|}{ Interrogativos y exclamativos } & SEGUNDA \\
\hline & & \multicolumn{2}{|c|}{ Con hiato acentual } & TERCERA \\
\hline & & \multirow{4}{*}{$\begin{array}{l}\text { No interrogativos } \\
\text { ni exclamativos y } \\
\text { sin hiato acentual }\end{array}$} & Agudos & CUARTA \\
\hline & & & Graves & QUINTA \\
\hline & & & Esdrújulos & SEXTA \\
\hline & & & Sobresdrújulos & SÉTIMA \\
\hline & \multicolumn{3}{|c|}{ Con dos acentos } & OCTAVA \\
\hline
\end{tabular}

Figura 4. Reglas que corresponden a los grupos terminales de palabras Fuente: Elaboración propia 
Lo anterior equivale a decir que la primera regla tiene como TOP los monosílabos con acento; la segunda, las palabras interrogativas y exclamativas; la tercera, las palabras con hiato acentual y así consecutivamente. Lo que sigue es señalar para cada regla su respectiva CT. La relación CT-TOP impide que el número de unidades de la CT sea superior al del TOP, por lo que no habrá reglas prohibitivas ni excepciones.

Entonces, la regla cuyo TOP son los monosílabos señala en su CT que se registran con tilde los pronombres /tu/, /mi/, /el/, /si/; los adverbios /si/ y /mas/; las formas verbales /de/ y /se/; las palabras interrogativas y exclamativas; $y$, finalmente, el nombre /te/ y su plural/tes/. No hay razón para formular una regla para tildar las nominalizaciones de estas palabras, debido a que ello es intrínseco a la función metalingüística del lenguaje: cualquier elemento lingüístico puede usarse como representante de sí mismo (Loureda, 2009).

La segunda regla, cuyo TOP son los polisílabos interrogativos y exclamativos, indica en su CT que a dichas palabras les corresponde tilde. Que el número de unidades de la CT coincida con el del TOP no infringe la coherencia de la norma. Algo similar ocurre con la tercera regla: en su TOP y en su CT están las palabras con hiato acentual. La cuarta norma, de palabras agudas en el TOP, indica en su CT que se tildan las que terminan en vocal, y vocal seguida de $<\mathrm{n}>$ o $<\mathrm{s}>$. La quinta, de las palabras graves como total, dice en su CT que se tilda las que culminan en secuencia consonántica y en cualquier consonante menos $<\mathrm{n}>\mathrm{y}$ $<\mathrm{s}>$. La CT de la sexta regla, de palabras esdrújulas en el TOP, indica que todas esas palabras se tildan. Por la CT de la séptima regla, se tildan las palabras sobresdrújulas, con lo cual se cubre todas las palabras de su TOP. Finalmente, la regla cuyo TOP son los adverbios terminados en -mente, dispone que se escribe con tilde aquellos cuyo primer constituyente fónico resulta comprendido en alguna CT luego de haber sido reingresado al SRAG.

\subsubsection{Agrupación de reglas}

Por la forma en que se relaciona con el sistema cognitivo del aprendizaje y con el SIPAE, la organización del SRAG no puede ser autónoma. Y el propósito de 
agrupar reglas es que se facilite su aprendizaje. El SRAG cuya organización reproduzca la del SIPAE sería más fácil de aprender, pues se trata del mismo patrón para ambos sistemas. Si los ordenamientos fueran diferentes se tendría que aprender dos patrones diferentes.

La unión de reglas exige cumplir dos requisitos: i) los TOP deben tener algo en común y las CT deben poder fusionarse; ii) la unión debe corresponder a alguna agrupación de terminales en el SIPAE. Así, no podrá unirse la sexta con la octava regla porque en el TOP de la sexta hay palabras esdrújulas, mientras que en el de la octava, unidades con dos acentos. A diferencia, existe compatibilidad entre las unidades del TOP de la segunda regla (graves interrogativas y exclamativas) y las del TOP de la quinta (graves no interrogativas ni exclamativas), pero hay incompatibilidad entre las CT correspondientes: la de la segunda regla establece que todas las palabras de su TOP van con tilde; la de la quinta, que solo las que terminan en secuencia consonántica y en cualquier consonante menos $<\mathrm{n}>\mathrm{y}<\mathrm{s}>$. La unión de estas dos reglas, daría lugar a excepciones.

Por lo señalado, el primer conjunto de reglas estaría constituido por la cuarta, quinta, sexta y séptima, pues sus TOP son compatibles. Estas normas toman palabras con el acento en las sílabas última, penúltima, antepenúltima y antes de la antepenúltima, respectivamente. En cuanto a las palabras comprendidas en sus CT, también hay compatibilidad: las terminaciones de las agudas a ser tildadas no se entrecruzan en absoluto con las terminaciones de las graves a ser tildadas y, según las CT de la sexta y la séptima, se tildan todas las palabras de su TOP. Además, esta unión se corresponde con una agrupación de palabras existente en el SIPAE.

En el caso de la segunda y la tercera regla, las unidades de sus TOP son diferentes: se trata de polisílabos graves interrogativos y exclamativos en la segunda y de palabras con hiato acentual en la tercera. Ambas CT comprenden a todas las palabras de sus TOP. Entonces, si las CT y los TOP son completamente diferentes la unión carece de sentido. Además, en el SIPAE estos dos grupos solo tienen en común ser polisílabos con un acento. Los motivos expuestos hacen también inviable la unión de la primera y la tercera regla. 
Tratándose de la primera y la segunda regla, sus TOP no se entrecruzan: en la primera, están todos los monosílabos; en la segunda, solo los graves interrogativos y exclamativos. En sus CT tampoco hay entrecruzamiento: en la primera se encuentran los monosílabos conocidos (mí, tú, quién...) y en la segunda, todas las palabras que aparecen en su TOP. Además, en los TOP y en las CT de ambas reglas hay palabras interrogativas y exclamativas. Esto favorece la unión porque ambos tipos de palabras pueden ser incluidas en un solo TOP y en una única CT; por tanto, se tendría lo siguiente: i) monosílabos, palabras interrogativas y exclamativas en el TOP; y ii) palabras interrogativas, exclamativas y los monosílabos de la lista que ya conocemos en la CT. Si bien esta unificación no se corresponde con algún agrupamiento de unidades en el SIPAE, tampoco lo violenta.

En las reglas tercera y octava, las palabras de sus TOP no se entrecruzan; hay palabras con hiato acentual en la tercera y palabras con dos acentos en la octava. Sus CT tampoco guardan alguna semejanza. Por tanto, la agrupación carece de sentido pues en el TOP y en la CT resultantes la información se mantendría separada.

Entonces, proponemos cuatro clases de tildes: i) diacrítica; ii) de hiato acentual, denominación justificada en otro trabajo (Mamani, 2013); iii) tópica, nombre también justificado (Mamani, 2013); iv) de reinicio, nombre que se sustenta en que su CT obliga a descomponer el adverbio, mantener en suspenso el sufijo -mente y someter el adjetivo respectivo al TOP que lo pudiese admitir y según ello evaluar si cumple con la CT respectiva. De ese modo, para decidir si se tilda /friamente/, nos quedamos con el adjetivo /fria/, el cual es un polisílabo con hiato acentual, por lo que va al TOP de la tilde de hiato acentual y según su CT le corresponde acento gráfico en la vocal cerrada. En el caso de /fasilmente/, el adjetivo /fasil/ es un polisílabo no interrogativo ni exclamativo y sin hiato acentual; por ello, le pertenece a la tilde tópica y, según su CT, debe tildarse por tratarse de una grave terminada en $<\mathrm{l}>$. Y en lo referente a $/ \mathrm{kruelmente/,} \mathrm{el} \mathrm{adjetivo} / \mathrm{kruel} / \mathrm{al}$ ser un monosílabo con acento está en el TOP de la tilde diacrítica y al no figurar en la lista de su CT, tendrá que escribirse sin acento escrito. Lo señalado puede verse en la figura 5 . 


\section{Discusión}

\subsection{Diferencias con la propuesta de la RAE y la ASALE}

Nuestra propuesta es muy diferente de la oficial. El TOP de la tilde diacrítica vigente está constituido por las siguientes palabras: i) los ocho monosílabos que ya se conoce (y "tés"); ii) las palabras interrogativas y exclamativas; y iii) las palabras átonas cuasihomónimas de las que conforman el grupo dos mencionado. La CT de esta tilde comprende a todas las unidades de su TOP, para indicar cuáles se tildan y cuáles no. En contraste, en el TOP de nuestra tilde diacrítica hay dos tipos de unidades: i) todos los monosílabos con acento; y ii) los polisílabos interrogativos y exclamativos. Y la CT de esta tilde incluye solo dos grupos de palabras: i) la lista de monosílabos ya conocidos; y ii) todas las palabras interrogativas y exclamativas (monosílabas y polisílabas).

En cuanto a la tilde tópica, su TOP está integrado por todos los polisílabos no interrogativos ni exclamativos y sin hiato acentual. Ello comprende a todas las esdrújulas y sobresdrújulas, y casi todas las agudas y graves. La CT de esta tilde engloba a todas las esdrújulas, sobresdrújulas, agudas terminadas en vocal o en vocal seguida de $<\mathrm{n}>\mathrm{o}<\mathrm{s}>$, y graves terminadas en secuencia consonántica y cualquier consonante menos $<\mathrm{n}>\mathrm{o}<\mathrm{s}>$. En el SRAG oficial lo que se parece a la tilde tópica son las reglas generales, pero el TOP de estas normas es vasto: i) todos los monosílabos que deja de lado la tilde diacrítica; ii) los polisílabos no interrogativos ni exclamativos; y iii) los polisílabos con dos acentos. En su CT, se puede identificar lo siguiente: i) todos los monosílabos de su TOP, pero para indicar que no les corresponde tilde; ii) las agudas que terminan en vocal o en vocal seguida de $<\mathrm{n}>\mathrm{o}<\mathrm{s}>$; iii) las graves que culminan en secuencia consonántica y en cualquier consonante menos $<\mathrm{n}>\mathrm{y}<\mathrm{s}>$; iv) todas las esdrújulas; v) todas las sobresdrújulas; vi) los polisílabos con hiato acentual, considerados aquí como excepciones; y vii) los adverbios culminados en -mente que se derivan de un adjetivo al cual le corresponde tilde.

Así, la organización del actual SRAG no encaja con el ordenamiento del SIPAE. Si la primera regla general señala que los monosílabos "no se acentúan nunca gráficamente, salvo en los casos de tilde diacrítica” (RAE \& ASALE, 2010, 
p. 231), entonces unos monosílabos se adscriben a las reglas generales y otros a la tilde diacrítica. Es decir, hay monosílabos en el TOP de las reglas generales $(<$ da $>,<$ di $>$, $<$ por $>$, etc.) y en el de la tilde diacrítica $(<$ dé $>,<$ sé $>$, $<$ quién $>$, $<$ dónde $>$, etc.). Para que este SRAG case con el sistema de palabras, habría que considerar los monosílabos sin acento, por lo cual estaríamos ante un sistema distinto del SIPAE, pues este no contiene tales unidades. En este hipotético sistema, las subdivisiones, en los monosílabos, serían las siguientes: i) por un lado estarán los que poseen acento $(<$ pan $>,<$ ron $>$, $<$ dé $>$, etc.) y por otro los que carecen de él $(<$ de $>,<$ quien $>,<\sin >$, etc. $)$; ii) entre los que tienen acento se debe apartar los interrogativos, exclamativos y los de la consabida lista ( $<$ dé $>,<$ él $>$, <quién $>$, etc.) del resto de monosílabos tónicos ( $<$ ron $>$, $<$ miel $>$, etc.); 3 ) entre los monosílabos sin acento se agrupará por un lado a los que no son cuasihomófonos de las palabras interrogativas y exclamativas $(<\sin >$, $<$ por $>,<\operatorname{con}>$, etc.) y por otro a los que sí son cuasihomófonos de esas palabras ( $<$ que $>$, <quien $>$, $<$ cual $>$, etc.).

Esta situación se complica más porque en la tilde diacrítica vigente, además de monosílabos, hay polisílabos; y la CT de esta tilde señala que los polisílabos interrogativos y exclamativos se tildan y que sus respectivos cuasihomófonos no. Entonces, el TOP de la tilde diacrítica no solo incluye a los polisílabos interrogativos y exclamativos, sino también a sus respectivos cuasihomófonos inacentuados. Ello obliga a realizar otra subdivisión en el grupo de los polisílabos: i) por un lado tendremos polisílabos con un acento; y ii) por otro, polisílabos sin acento entre los cuales estarán los cuasihomófonos señalados. Esta relación entre el SRAG imperante y el sistema de palabras se puede apreciar en la figura 6. 


\begin{tabular}{|c|c|c|c|c|c|}
\hline \multicolumn{4}{|c|}{ Organización de palabras } & \multicolumn{2}{|c|}{ Clases de tilde } \\
\hline \multirow{4}{*}{ Monosílabas } & \multirow{2}{*}{ Sin acento } & \multicolumn{2}{|c|}{ No cuasi homófonas de las interrogativas y exclamativas } & & \multirow{6}{*}{ Reglas } \\
\hline & & \multicolumn{2}{|c|}{ Cuasi homófonas de las interrogativas y exclamativas } & \multirow{5}{*}{ Diacrítica } & \\
\hline & \multirow{2}{*}{ Con acento } & \multicolumn{2}{|c|}{ Interrogativas, exclamativas y las ocho de la lista } & & \\
\hline & & \multicolumn{2}{|c|}{ No cuasi homófonas de las interrogativas y exclamativas } & & \\
\hline \multirow{8}{*}{ Polisílabas } & Sin acento & Cuasi homófonas de las i & sy exclamativas & & \\
\hline & \multirow{6}{*}{$\begin{array}{l}\text { Con un } \\
\text { acento }\end{array}$} & \multicolumn{2}{|c|}{ Interrogativas y exclamativas } & & \\
\hline & & \multirow{4}{*}{$\begin{array}{l}\text { Ni interrogativas ni } \\
\text { exclamativas y sin hiato } \\
\text { acentual }\end{array}$} & Agudas & \multirow{6}{*}{\multicolumn{2}{|c|}{ generales }} \\
\hline & & & Graves & & \\
\hline & & & Esdrújulas & & \\
\hline & & & Sobresdrújulas & & \\
\hline & & \multicolumn{2}{|c|}{ Con hiato acentual } & & \\
\hline & \multicolumn{3}{|c|}{ Con dos acentos } & & \\
\hline
\end{tabular}

Figura 6. Relación entre las clases de tildes de la RAE y la ASALE, y la organización de palabras Fuente: Elaboración propia

Esta falta de correspondencia se debería a que en la creación del SRAG, y posteriores modificaciones, no se habría considerado que las palabras conforman un sistema. Una de las consecuencias de ello es que se sobrevalora el rol de la cantidad de palabras comprendidas en las reglas, lo que da lugar a la creación de las reglas generales. Esta visión tampoco percibe como una contradicción la existencia de reglas prohibitivas, a pesar de que violentan los siguientes principios y funciones instituidos por la RAE y la ASALE (2010): i) principio B (la función del SRAG no es distinguir entre palabras tónicas y átonas); ii) principio $\mathrm{K}$ (de economía); iii) función prosódica (la tilde señala la sílaba acentuada); y iv) función diacrítica (la tilde distingue la forma tónica de la átona). En el caso de las palabras con dos acentos, se habría decidido por su descomposición morfológica porque no se considera que el SRAG se aplica a la palabra escrita (SIPAE) y que esta se basa en la palabra morfosintáctica (SIPACA).

\subsection{Palabra escrita y palabra morfosintáctica}

Considerar que la palabra escrita se estructura sobre la palabra morfosintáctica brinda fundamento para haber suprimido la regla por la que se escribían con tilde los verbos con enclíticos si tenían esta marca antes de la adjunción del clítico: 
"murióse", “déme", "sélo", “deténte”, etc. (Ramírez, 1993). Esta norma era contraproducente con la noción "palabra escrita", ya que obligaba a la descomposición morfológica de dicha unidad.

Suponemos que ignorar que la tilde se aplica a la palabra escrita ha causado que en el actual SRAG se haya dispuesto que 'tés', se registre con tilde. Si la base de la palabra escrita es la palabra morfosintáctica, entonces "té" es una palabra distinta de "tés". La lasitud en este aspecto puede conducir a que en el futuro se pueda admitir la tilde en 'qués', plural de 'qué' nominalizado, y así consecutivamente. Además, si la justificación para tildar "tés" es la probable confusión con el plural del nombre de la letra "te", sería inconsistente pues al tener distinto género gramatical sus contextos son muy diferentes.

Asimismo, la descomposición morfológica de los adverbios en -mente a efectos de la tilde cuestiona su estatus de palabras: el adjetivo es una palabra distinta del adverbio resultante de la combinación con -mente.

\subsection{Algunas consecuencias de nuestra propuesta}

\subsubsection{Conocer las palabras antes que el SRAG}

Si el ordenamiento del SRAG está determinado por la organización del SIPAE (que se organiza según el SIPACA), este debe dominarse plenamente antes que el SRAG. Nuestra propuesta sirve incluso para señalar la secuencia: la oposición polisílabo/monosílabo se aprenderá primero. Luego se debe saber distinguir entre polisílabos con un acento y polisílabos con dos acentos. Posteriormente, de entre los polisílabos con un acento se debe diferenciar entre tres grupos: i) interrogativos y exclamativos; ii) palabras con hiato acentual; iii) polisílabos no interrogativos ni exclamativos y sin hiato acentual. Finalmente, dentro de los polisílabos no interrogativos ni exclamativos y que no contengan hiato acentual, se debe realizar una distinción de las palabras por el lugar del acento (véase la figura 3).

\subsubsection{La enseñanza del SRAG}

Tras haber aprendido la organización del SIPAE, se emparejará cada grupo termi- 
nal de palabras con las clases de tildes propuestas. Ello significa que después de exponer en qué consiste el acento escrito se tendrá de señalar que existen cuatro clases de tildes y enseguida limitarse a indicar el TOP de cada clase. Con esta simple asociación, el aprendiz sabrá que a cada clase de tilde le corresponde un conjunto de palabras con características específicas.

El siguiente paso implica decidir por cuál clase de tilde empezar. Para ello se requiere considerar la información de cada $\mathrm{CT}$, pues allí se exponen los requisitos que deben satisfacer las palabras para poder ser escritas con acento gráfico. La CT con más especificaciones será más difícil de aprender porque se procesa una mayor cantidad de información. Como la única clase de tilde que en su CT tiene las mismas palabras que en su TOP es la tilde de hiato acentual, esta será la primera en enseñarse. Con este mismo criterio, la siguiente clase de tilde a impartir sería la tópica, pues para las esdrújulas y sobresdrújulas los TOP y las CT respectivos comprenden las mismas unidades; en las agudas, la CT incluye las que terminan en vocal y en vocal seguida $\mathrm{de}<\mathrm{n}>\mathrm{o}<\mathrm{s}>$; y la CT de las graves solo abarca a las que culminan en cualquier secuencia consonántica y en cualquier consonante que no sean $<\mathrm{n}>\mathrm{o}<\mathrm{s}>$. Se seguirá con la tilde diacrítica, pues su CT contiene las palabras interrogativas y exclamativas; el nombre "té" y su plural; los pronombres "mí", "tú”, “él”, "sí”; las formas verbales "sé” y "dé”; los adverbios "sín" y "más". La última tilde a abordar será la de reinicio ya que su CT supone la descomposición de la palabra, la reubicación del adjetivo en cualquiera de los tres TOP anteriores y el cumplimiento de los requisitos de la CT que correspondiese.

\subsubsection{Rendimiento de principios}

Respecto del ordenamiento de reglas vigente, el propuesto guarda mayor coherencia con el principio A del acento escrito (RAE \& ASALE, 2010) (solo las palabras tónicas son susceptibles de ser tildadas) porque ni siquiera ingresan al sistema las palabras átonas. Asimismo, al no tener reglas prohibitivas, cumple en mayor grado el principio B (la función de la tilde no es distinguir entre palabras tónicas y átonas). Finalmente, se maximiza el principio K (se empleará el menor número de tildes), aunque aplicado al número de reglas. 


\subsubsection{Diferentes tipificaciones de errores}

Si un hablante que inicia sus estudios universitarios escribe "No sé por que se fué Andrés", según lo propuesto, solo ha infringido nuestra tilde diacrítica, por lo cual en la corrección se trabajará la identificación de los monosílabos (parte del TOP) y dentro de ese grupo a cuáles les corresponde acento gráfico (según la CT respectiva). En cambio, para la RAE y la ASALE, se trataría de equivocaciones tanto en la tilde diacrítica (por "qué") como en las reglas generales (por "fue"); por consiguiente, en la corrección se deberá realizar dos acciones: i) reforzar la idea de que por regla general los monosílabos no se tildan, pero excepcionalmente algunos sí, por la tilde diacrítica; ii) reforzar las reglas de la tilde diacrítica. Asimismo, si otro hablante del mismo nivel de instrucción escribe "El alcaide sentia lástima por Dario", en nuestra óptica, ha incurrido en un error focalizado en la tilde de hiato acentual. Por lo tanto, la corrección se limitará a señalar que estas palabras están en el dominio de la tilde de hiato acentual razón por la cual se tilda la vocal cerrada de dicha secuencia. Pero en la propuesta oficial, se trata de un problema en las excepciones de las reglas generales; por lo tanto, se optará por lo siguiente: i) se tendrá que admitir que esas palabras son graves terminadas en vocal; ii) se deberá señalar que tales vocablos se tildan por ser casos excepcionales al presentar hiato acentual.

\subsubsection{Las palabras de dos acentos}

Para tratar los adverbios culminados en -mente se dispone de tres alternativas: i) tildarlos todos; ii) tildar algunos; iii) no tildar ninguno. Cualquiera de estas tres posibilidades puede expresarse en la CT de la regla respectiva. Sería preferible evitar la tercera, porque ello supondría una regla prohibitiva. Como se conoce, la RAE y la ASALE han optado por la segunda y la forma en que lo han hecho violenta la relación SRAG-SIPAE, ya que cuestiona su estatus de palabras al descomponerlos en dos partes. Si se sigue la primera alternativa, debido a que una palabra solo puede tener una tilde, se debe decidir si esta representará la primera sílaba tónica o la segunda. Optar por la tilde en la segunda sílaba tónica respeta la condición de palabras que tienen estas unidades, pero genera dos inconvenientes: 
i) contraviene la regla de las palabras graves que terminan en vocal; y aunque se pueda argüir que estos adverbios no son propiamente palabras llanas por poseer dos acentos, ello no anula el que estas palabras presenten intensidad en la penúltima sílaba; y ii) se carecería de fundamento para no haber tildado la primera sílaba tónica: cualquier justificación que se proporcione en este sentido será artificial.

Si se decide tildar la primera sílaba tónica, la CT de esta regla expresaría: "Se tilda la primera sílaba tónica de las palabras que tienen dos acentos". Esta norma tiene las siguientes virtudes: i) no hace alusión a ninguna otra regla; ii) respeta el estatus de palabra de estas unidades; iii) no violenta la relación entre los sistemas implicados; 4) respeta uno de los principios del SRAG que es el de marcar el acento; y 5) no distorsiona las CT de otras reglas con las que guarda cierta semejanza (no se tildan las palabras graves que terminan en vocal y se tildan las esdrújulas y sobresdrújulas).

Es posible conseguir que estas palabras se tilden sin tener que recurrir a una tilde ad hoc, condición que ostenta la denominada tilde de reinicio. Se puede eliminar la tilde de reinicio, pero ello no conduce automáticamente a la desaparición de la octava regla; es más, esta norma no puede ser anulada porque es por ella que las palabras con dos acentos logran ingresar al SRAG. Si consideramos que la nueva CT de la octava regla establece que se tilda la primera sílaba tónica de las palabras con dos acentos, nos podemos percatar de que tiene semejanzas con las CT de las reglas de la tilde tópica, razón por la cual se puede plantear la fusión de la octava regla con las reglas de la tilde tópica. Con esta unión, como ya se sabe, no se puede alterar en lo más mínimo los TOP de cada regla, pero sí se pueden realizar modificaciones en las CT respectivas. Entonces, lo que proponemos es lo siguiente: i) mantener inalterable la CT que corresponde a las palabras agudas; ii) en el caso de la CT de las palabras graves solo se indicaría que se tildan las palabras que presentan acento en la penúltima sílaba si terminan en secuencia consonántica y en cualquier consonante menos $<\mathrm{n}>\mathrm{o}<\mathrm{s}>$. Como se puede notar, en esta CT no solo están incluidas las palabras llanas sino también los adverbios que terminan en -mente. Para el resto de palabras, solo se indicaría que se escribirán con tilde aquellas que presenten acento en la antepenúltima o antes de la 
antepenúltima sílaba. Es evidente que aquí están comprendidas las esdrújulas, sobresdrújulas y todos estos adverbios.

\section{Conclusiones}

A pesar de la denominación, el actual SRAG no cuenta con reglas generales. Por ello, su enseñanza no puede iniciarse con la tilde en las palabras agudas, graves, esdrújulas y sobresdrújulas.

El ordenamiento del SRAG propuesto sería más fácil de aprender que el actual por lo siguiente: i) guarda coherencia con la forma en que están organizados el SIPAE y el SIPACA; ii) no presenta reglas prohibitivas ni excepciones; y iii) no presenta asimetrías.

La base sobre la que se erige la organización planteada permite proponer secuencias en la enseñanza, tanto del SIPAE como de las normas del acento gráfico.

El aprendizaje del SRAG exige seguir la siguiente sucesión general: i) identificar cada uno de los grupos de palabras; ii) asignar cada grupo terminal de palabras (como su TOP) a cada clase de tilde; y iii) dominar la información contenida en la CT de cada clase de tilde.

Respecto del aprendizaje del SIPAE y el SIPACA, se debe determinar primero si la palabra es un monosílabo o un polisílabo; luego, si es un polisílabo, debe establecerse si tiene uno o dos acentos; después, de entre los polisílabos con un acento se debe determinar si tienen o no hiato acentual; posteriormente, se reconocerá las palabras interrogativas y exclamativas; luego, de entre las que no contienen hiato acentual ni son interrogativas ni exclamativas se distinguirá entre agudas, graves, esdrújulas y sobresdrújulas.

\section{Sugerencias}

Para enseñar el acento escrito, habría que considerar por lo menos tres aspectos: i) los conocimientos metalingüísticos implicados (Mamani, 2013); ii) cómo están organizados el SIPAE y el SIPACA; y iii) cómo está organizado el SRAG. Si tenemos en cuenta que lo abordado en nuestro trabajo supone la relación lengua- 
escritura, es probable que se necesite considerar estos tres factores en la enseñanza de los otros ámbitos de la ortografía.

Si cualquier unidad lingüística (palabra, sílaba, frase, oración) puede representarse a sí misma, su identidad no puede ser alterada al producirse esta operación. Por tanto, si la oración "qué dirán" va a ser objeto de referencia, la nominalización respectiva debe respetar tal estatus. Es decir, en tal uso tendrá que escribirse "qué dirán" y no "quédirán”. Optar por lo último significa apartarse de lo que ocurre en el sistema lingüístico y generar, además, serios problemas para la tilde porque esa "única" palabra presenta dos acentos, por lo cual se tendría que crear otra regla para indicar cómo proceder en tal caso. Si se respeta el estatus de la unidad nominalizada no se requiere de ninguna regla adicional. Ser coherentes con esta perspectiva permite afirmar que la nominalización de la frase preposicional "por qué" no puede dar como resultado la palabra "porqué". De continuarse con este proceder, se tendría "quédirán" como nominalización de "qué dirán" o "paraqué" de "para qué".

Al decidir sobre la escritura de las unidades complejas, no se puede ignorar la unidad lingüística de referencia, esto es, la palabra morfosintáctica. Manejar con coherencia los criterios con que se decide si algún compuesto se escribe con guion o sin él podría permitir relacionar la escritura, por ejemplo, de "históricocrítico", con la de los adverbios que culminan en -mente, pues ambas unidades tienen dos acentos.

\section{Referencias bibliográficas}

Arnold, M. \& Osorio, F. (1998). Introducción a los conceptos básicos de la teoría general de sistemas. Cinta Moebio, 3, 40-49.

Baicchi, A. (2015). Construction Learning as a Complex Adaptative System. Nueva York: Springer.

Beaugrande, R. de \& Dressler, W. (2005). Introducción a la lingüística del texto. Barcelona: Ariel.

Duval, G. (2014). Teoría de sistemas. Una perspectiva constructivista. En S. Ramírez (Coord.), Perspectivas en las teorías de sistemas (pp. 75-82). Ciu- 
dad de México: Universidad Nacional Autónoma de México, Centro de Investigaciones Interdisciplinarias en Ciencias y Humanidades.

Earls, J. (2011). Introducción a la teoría de sistemas complejos. Lima: Pontificia Universidad Católica del Perú.

Echeverría, R. (2010). El observador y su mundo (Vol. II). Buenos Aires: Granica. García, R. (2006). Sistemas complejos. Conceptos, método y fundamentación epistemológica de la investigación interdisciplinaria. Barcelona: Gedisa.

García, R. (2011). Interdisciplinariedad y sistemas complejos. Revista Latinoamericana de Metodología de las Ciencias Sociales, 1(1), 65-101.

Hualde, J. \& Colina, S. (2014). Los sonidos del español. Nueva York: Cambridge University.

López, J. (2013). Aplicación del marco de los sistemas complejos adaptativos a un modelo de variación lingüística. Moenia, 19, 5-24.

Loureda, O. (2009). De la función metalingüistica al metalenguaje: los estudios sobre el metalenguaje en la lingüistica actual. Revista Signos. Estudios de Lingüística, 42(71), 317-332.

Mamani, L. (2013). Conocimientos metalingüisticos y uso correcto de la tilde. Revista Signos. Estudios de Lingüística, 46(83), 389-407.

Martínez, A. (2010). El problema del cambio, desde la diacronía al sistemismo. Recuperado de: http://www.biblioteca.org.ar/libros/154338.pdf.

Minedu. (2009). Diseño curricular nacional. Lima, Perú: Ministerio de Educación. Recuperado de: www.minedu.gob.pe/DeInteres/xtras/download. php?link=den_2009.pdf.

Mineduc. (2012). Bases curriculares. Lenguaje y Comunicación. Santiago, Chile: Ministerio de Educación de Chile. Recuperado de: http://www.curriculumenlineamineduc.cl/605/articles-21322_bases.pdf

Mineduc. (2016). Bases curriculares. $7^{\circ}$ básico a $2^{\circ}$ medio. Santiago, Chile: Ministerio de Educación de Chile. Recuperado de: http://www.curriculumenlineamineduc.cl/605/articles-37136_bases.pdf

Ministerio de Educación de Ecuador. (2016). Currículo de Educación General Básica y Bachillerato General Unificado de Lengua y Literatura. s. d. Recuperado de https://educacion.gob.ec/wp-content/uploads/downloads/2016/03/LENGUA.pdf

Moreno, F. (2008). Principios de sociolingüistica y sociología del lenguaje. Barcelona: Ariel. 
Núñez, R. \& Morales-Front, A. (1999). Fonología contemporánea de la lengua española. Washington D. C.: Georgetown University Press.

Portilla, L. (2003). El problema de la acentuación ortográfica de los estudiantes sanmarquinos. Recuperado de http://www.cybertesis.edu.pe/sisbib/2003/portilla_dl/pdf/portilla_dl.pdf

Pujol, M. (2005). La ortografía. En S. Torner \& P. Battaner (Eds.), El corpus PAAU 1992: Estudios descriptivos, textos y vocabulario (pp. 29-65). Barcelona: Institut Universitari de Lingüística Aplicada.

Radford, A., Atkinson, M., Britain, D., Clahsen, H., \& Spencer, A. (2000). Introducción a la lingüística. Madrid: Cambridge University Press.

RAE \& ASALE. (2010). Ortografía de la lengua española. Madrid: Espasa.

RAE \& ASALE. (2011). Nueva gramática de la lengua española. Fonética y fonología. Madrid: Espasa.

Ramírez, L. (1993). El acento escrito. Fundamentos teóricos y lingüísticos. Lima: Universidad Nacional Mayor de San Marcos.

Ramírez, S. (2014). Perspectivas en las teorías de sistemas. Ciudad de México: Universidad Nacional Autónoma de México, Centro de Investigaciones Interdisciplinarias en Ciencias y Humanidades.

Rodríguez, L. (2011). Exploraciones de la complejidad. Aproximación introductoria al pensamiento complejo y a la teoría de los sistemas complejos. Buenos Aires: Centro Iberoamericano de Estudios en Comunicación, Información y Desarrollo.

Silva-Corvalán, C. (2001). Sociolingüistica y pragmática del español. Washington D. C.: Georgetown University Press. 